\title{
Cervical carcinoma high-expressed long non-coding RNA 1 promotes papillary thyroid carcinoma cell proliferation and invasion
}

\author{
Ping Shi ${ }^{1}$, Yan Liu ${ }^{1}$, Mingzeng Zhang ${ }^{2}$, Jianwang Yang ${ }^{3}$, Shanghua Jing ${ }^{1}$, Dongqiang Yang ${ }^{4}$, Fei Liu ${ }^{5}$, \\ Yanzhao $\mathrm{Wu}^{1}$, Huijing Shi ${ }^{1}$, Cuizhi Geng ${ }^{6}$ \\ ${ }^{1}$ Department of Otolaryngology Head and Neck Surgery, Hebei Medical University Fourth Affiliated Hospital, Shijiazhuang, China; ${ }^{2}$ Department of \\ Hematology, Hebei Medical University Fourth Affiliated Hospital, Shijiazhuang, China; ${ }^{3}$ Department of Scientific Research Center, Hebei Medical \\ University Second Affiliated Hospital, Shijiazhuang, China; ${ }^{4}$ Department of Radiological Intervention, Hebei Medical University Fourth Affiliated \\ Hospital, Shijiazhuang, China; ${ }^{5}$ Department of Scientific Research Center, Hebei Medical University Fourth Affiliated Hospital, Shijiazhuang, \\ China; ${ }^{6}$ Department of General Surgery, Hebei Medical University Fourth Affiliated Hospital, Shijiazhuang, China \\ Contributions: (I) Conception and design: P Shi, C Geng; (II) Administrative support: S Jing, H Shi, C Geng; (III) Provision of study materials or \\ patients: S Jing, Y Wu, H Shi; (IV) Collection and assembly of data: P Shi, Y Liu, D Yang; (V) Data analysis and interpretation: P Shi, M Zhang, J \\ Yang, F Liu; (VI) Manuscript writing: All authors; (VII) Final approval of manuscript: All authors. \\ Correspondence to: Cuizhi Geng. Department of General Surgery, Hebei Medical University Fourth Affiliated Hospital, Shijiazhuang, China. \\ Email: geng0923@163.com.
}

Background: Studies have shown that cervical carcinoma high-expressed long non-coding RNA 1 ( IncRNA-CCHE1) may promote tumor development by regulating tumor migration and invasion in a variety

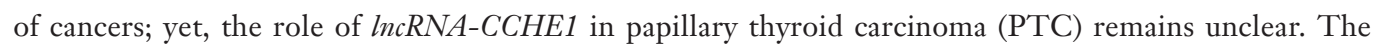

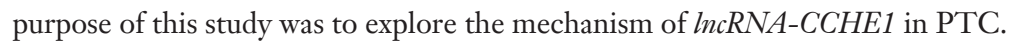

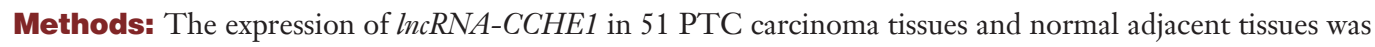
measured using real-time quantitative polymerase chain reaction (RT-qPCR). Cell Counting Kit-8 (CCK8),

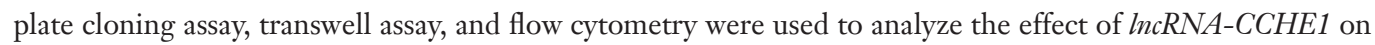
PTC cell proliferation, invasion, and apoptosis in vitro.

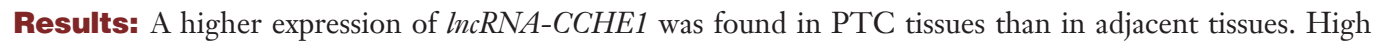

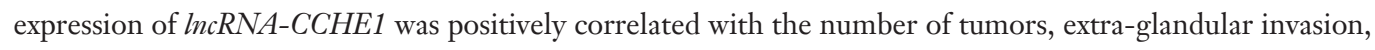

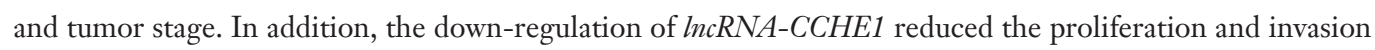
of PTC cell lines and promoted cell apoptosis, while its up-regulation caused the opposite effect. These effects were regulated via the extracellular signal-regulated kinase/mitogen-activated protein kinase (ERK/ MAPK) pathway.

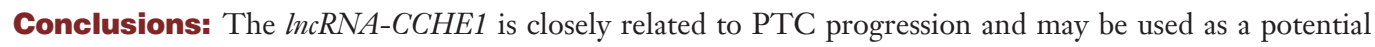
biomarker for early diagnosis and treatment of PTC.

Keywords: Cervical carcinoma high-expressed long non-coding RNA 1 (IncRNA-CCHE1); papillary thyroid carcinoma (PTC); cell proliferation; early diagnoses; extracellular signal-regulated kinase/mitogen-activated protein kinase (ERK/MAPK)

Submitted Jul 16, 2021. Accepted for publication Aug 27, 2021.

doi: $10.21037 /$ tcr-21-1502

View this article at: https://dx.doi.org/10.21037/tcr-21-1502 


\section{Introduction}

The incidence of thyroid carcinoma (TC) has rapidly increased over recent years. Currently, TC is the fourth most common female malignancy. Papillary TC (PTC) accounts for $80-85 \%$ of thyroid cancers (1). Compared with other TC types, PTC is less malignant, but it also has a higher incidence of distant metastasis and higher recurrence rates. In addition, not all types of PTC are associated with long survival (2). For these patients how to choose a reasonable and effective surgical type is critical. The main controversies are related to the timing of preventive central or lateral neck dissection. The 2015 American Thyroid Association (ATA) guidelines (3) do not recommend fine needle aspiration (FNA) for all grades of clinical stage PTC patients. Some biochemical characteristics of papillary thyroid carcinoma (PTC), such as thyroid stimulating hormone (TSH), thyroglobulin (TG), thyroid peroxidase antibody (TPOAb), and so on, was usually just used to monitor the follow-up and prognosis of the disease after operation (3). In addition, considering that a complete and accurate clinical judgment cannot be made based only on ultrasound. Therefore, the search for potential molecular biomarkers, as an important preoperative evaluation index to recognize the tumor with more aggressive, is of crucial importance for clinicians to choose positive type of operation.

Long non-coding RNAs (lncRNAs) are transcripts with lengths exceeding 200 nucleotides, with no protein-coding capacity found in the nucleus or cytoplasm (4), and they lack an open reading frame $(5,6)$. Half of the human genome is made of lncRNAs; nevertheless, the function of most lncRNAs is unknown (7). Recent studies have demonstrated that IncRNA expression may have a crucial role in tumor occurrence and development (8-10). For example, $\operatorname{lnc} R N A$ $M A L A T 1$ has been suggested to act as a tumor promoter in hepatocellular carcinoma (11), colorectal cancer (12), and cervical cancer (13). The IncRNA ADAMTS9-AS2 can act as a tumor suppressor gene in a wide variety of tumors, including gastric cancer (14), ovarian cancer (15), and breast cancer (16). Cervical carcinoma high-expressed lncRNA 1 ( $\ln R \mathrm{RNA}$-CCHE1) has received increasing attention over recent years. Previous studies have shown that $\ln R N A$ CCHE1 may promote tumor development by regulating tumor migration and invasion in a variety of cancers (17-20). It has been reported to be highly expressed in cervical cancer tissues and has been suggested as a potential prognostic molecular marker of cervical cancers (21).

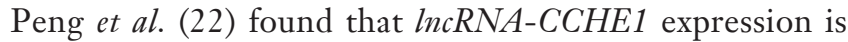

correlated with poor prognosis in hepatocellular carcinoma,

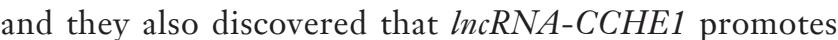
carcinogenesis through activation of the extracellular signalregulated kinase/mitogen-activated protein kinase (ERK/ MAPK) pathway.

The hypothalamus-pituitary secretory axis regulates the thyroid gland and uterus. Some academics believe that there is an internal relationship between thyroid cancer and cervical disease (23-25). However, the function of IncRNA-CCHE1 in PTC has rarely been reported, and the relationship between its expression and the patient's clinicopathological characteristics has not been certain. The purpose of this study was to explore the mechanism of

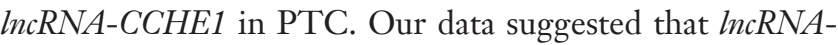
CCHE1 may be used as a potential biomarker for early diagnosis in patients with PTC. We present the following article in accordance with the MDAR reporting checklist (available at https://dx.doi.org/10.21037/tcr-21-1502).

\section{Methods}

\section{Tissue samples}

A total of 51 sample pairs from primary diagnosed PTC from patients undergoing surgery at the Hebei Medical University Fourth Affiliated Hospital between August 2018 and June 2019 (Hebei Province, China) were collected and reviewed. Each sample pair included normal and cancerous tissue of the same patient. Clinical data, including age, gender, tumor size, tumor invasion, tumor number, lymph node metastasis, pathological grade, and BrafV600E mutation, were collected from all participants.

All procedures performed in this study involving human participants were in accordance with the Declaration of Helsinki (as revised in 2013). This study was approved by the Ethics Committee of Hebei Medical University Fourth Affiliated Hospital (2020KS018). All participants fully understood the experimental protocol and signed informed consent forms. Patient consent for publication is not applicable.

\section{Cell culture}

The human PTC cell lines TPC-1 and BCPAP were purchased from Shanghai Zhong Qiao Xin Zhou Biotechnology Co., Ltd. (Shanghai, China). The cell lines K1 (source of PTC) and FTC-133 (source of thyroid follicular carcinoma) were a kind gift from the Sichuan 
University Research Center.

All cell lines were cultured in Roswell Park Memorial Institute (RPMI) 1640 medium (Shanghai Zhong Qiao Xin Zhou Biotechnology Co., Ltd., Shanghai, China) supplemented with $10 \%$ fetal bovine serum (FBS; Gibco, Waltham, MA, USA) in a humidified atmosphere containing $5 \% \mathrm{CO}_{2} / 95 \%$ air at $37^{\circ} \mathrm{C}$.

\section{Real-time quantitative polymerase chain reaction (RT-qPCR)}

Total RNA was extracted using Trizol (Thermo Fisher Scientific, Waltham, MA, USA), and circular DNA (cDNA) was synthesized by reverse transcription. The RT-qPCR reaction conditions were as follows: $5 \mathrm{~min}$ at $95^{\circ} \mathrm{C}$ for predenaturation, then $30 \mathrm{~s}$ at $95^{\circ} \mathrm{C}, 30 \mathrm{~s}$ at $60^{\circ} \mathrm{C}$, and $30 \mathrm{~s}$ at $72^{\circ} \mathrm{C}$, for a total of 40 cycles. The primer sequences were as follows: IncRNA-CCHE1: 5'-AAGGTCCCAGGATACTCGC-3' (forward) and 5'-GTGTCGTGGACTGGCAAAAT-3' (reverse). The internal control GAPDH primer was: 5'-CGCTGAGTACGTCGTGGAGTC-3' (forward): 5'-GCTGATGATCTTGAGGCTGTTGTC-3' (reverse). The results were calculated with $2^{-\Delta \Lambda C T}$.

\section{Transfection}

Cells over-expressing $\ln R N A$-CCHE1 (lnc CCHE1) and

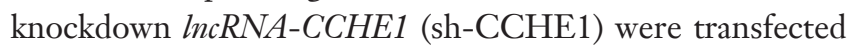
and non-sense in lentiviral vectors (GenePharma, Shanghai, China). Cell transfection was performed using lipofectamine 2000 (Invitrogen, Carlsbad, CA, USA), following the manufacturer's instructions. Cells were collected $24 \mathrm{~h}$ after transfection.

\section{Cell Counting Kit-8 (CCK-8) assay}

Cell proliferation was assessed using the CCK-8 method. Briefly, BCPAP cells were seeded in 96-well plates at a concentration of 1,500 cells/well. Cells were cultured for 24, 48 , and $72 \mathrm{~h}$. At each time point, $10 \mu \mathrm{L}$ of sterile CCK-8 was added to each well and incubated for another $4 \mathrm{~h}$ at $37^{\circ} \mathrm{C}$. The absorbance optical density (OD) at $450 \mathrm{~nm}$ was determined using a microplate reader (Anthos, Salzburg, Austria).

\section{Colony formation assay}

A colony formation assay was used to evaluate the proliferation of PTC cells. The BCPAP cells were digested using trypsin and cultured in 6-well plates. An average of
1,000 cells was seeded per well in a medium containing $10 \%$ FBS. After culturing for 2-3 weeks, colony formation was analyzed; the colony formation rate was calculated using the following formula: colony formation rate $=$ number of colonies/number of seeded cells $\times 100 \%$.

\section{Transwell assay}

A transwell assay was used to evaluate the invasion ability of PTC cells. After transfection for $24 \mathrm{~h}$, cells were digested by trypsin. The PTC cells in each group were suspended in a serum-free medium, and the concentration was adjusted to $2 \times 10^{5}$ cells/well. A volume of $200 \mu \mathrm{L}$ of cell suspension was added to the transwell plate's upper chamber and $10 \%$ FBS to the lower chamber. Cells were cultured for $60 \mathrm{~h}$, after which cells at the bottom of the chamber were fixed and stained with crystal violet for $20 \mathrm{~min}$, followed by air-drying. The numbers of cells in five random fields of view (FOV) were counted under a microscope (Nikon, Shinagawa, Tokyo, Japan), and the differences between the groups were compared. We selected five random FOV and the images were captured under a microscope at a magnification of $\times 20$.

\section{Cell apoptosis}

The $1 \times 10^{5}-5 \times 10^{5}$ cells were washed 3 times with phosphate-buffered saline (PBS) and suspended in $100 \mu \mathrm{L}$ binding buffer, and then with a $400 \mu \mathrm{L}$ binding buffer containing $5 \mu \mathrm{L}$ propidium iodide (PI; Roche, Shanghai, China). Samples were then mixed with $5 \mu \mathrm{L}$ actin $\mathrm{V}$ (Roche, Shanghai, China) in the dark for $15 \mathrm{~min}$ at room temperature. Apoptosis was measured by flow cytometry (Beckman Coulter, Brea, CA, USA).

\section{Western blotting}

Harvested cells $\left(2 \times 10^{6}\right)$ were placed into $1.5 \mathrm{~mL}$ Eppendorf tubes and mixed with cell lysate $[99 \mu \mathrm{L}$ cell lysate $+1 \mu \mathrm{L}$ phenylmethylsulfonyl fluoride (PMSF)] and incubated on ice for $30 \mathrm{~min}$. They were then centrifuged at 3,500 rpm for $30 \mathrm{~min}$ at $4{ }^{\circ} \mathrm{C}$, the liquid (total protein) was carefully removed into another EP tube. Western blot was performed using a $10 \%$ sodium dodecyl sulfate-polyacrylamide gel electrophoresis (SDS-PAGE). After separations, the proteins were transferred to a polyvinylidene fluoride (PVDF) membrane. Membranes were blocked with 5\% skimmed milk at $37^{\circ} \mathrm{C}$ for $1 \mathrm{~h}$. Membranes were incubated 

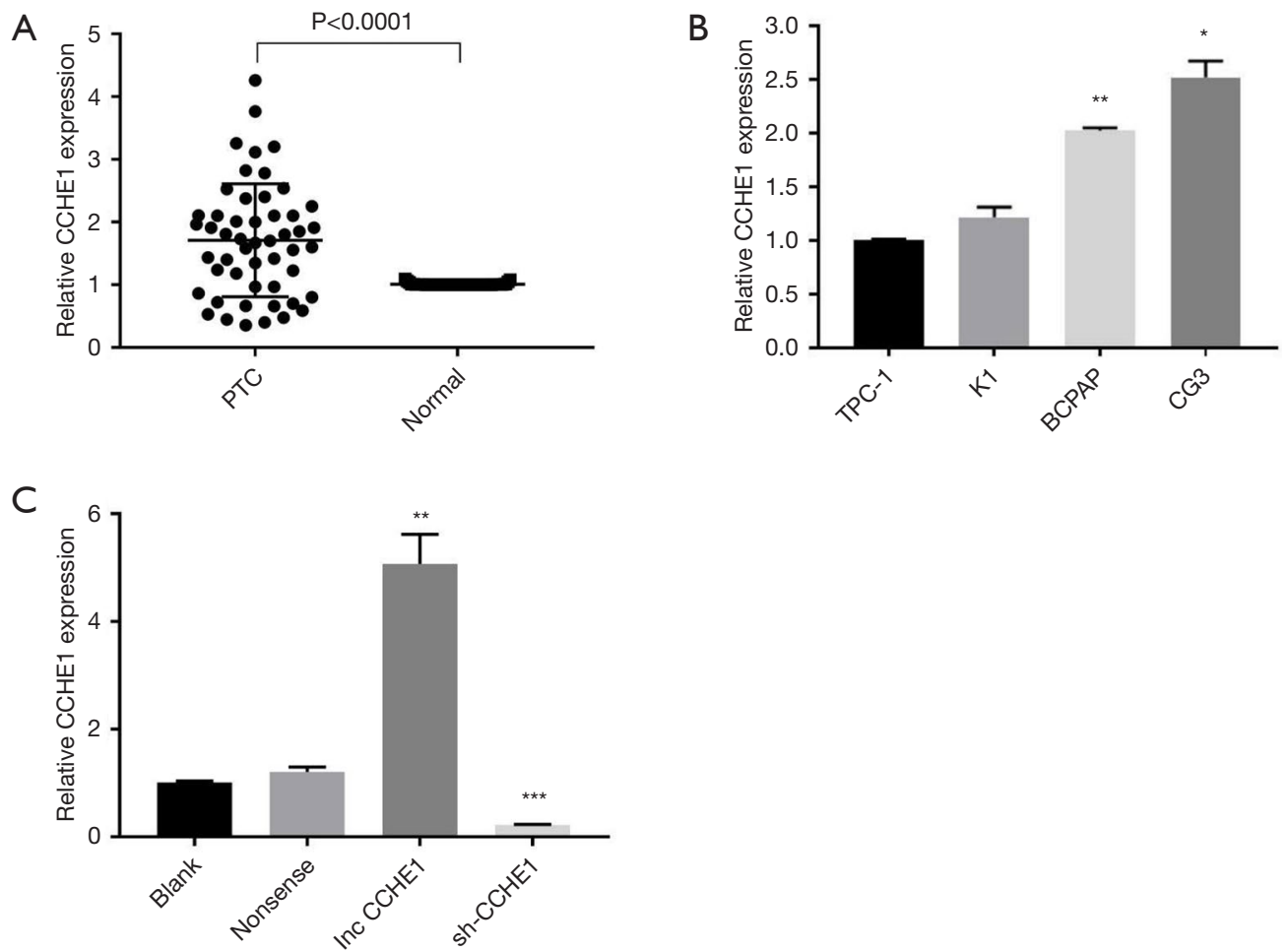

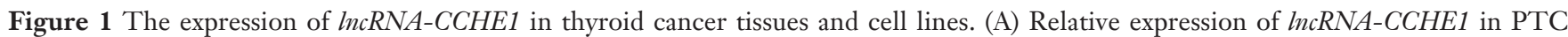
tissues and adjacent normal analyzed by RT-qPCR. (B) LncRNA-CCHE1 expression in PTC cell lines. (C) Compared with the non-sense

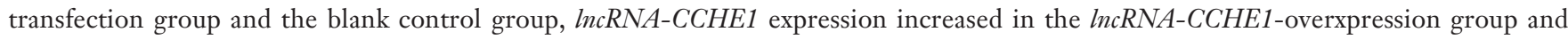
decreased in the $\ln R$ RNA-CCHE1-knockdown group. Data in $(\mathrm{B}, \mathrm{C})$ are means \pm SDs of three independent experiments using $\mathrm{PTC}$ cells from different HDs. *, $\mathrm{P}<0.05 ;$ ** $\mathrm{P}<0.01$; ${ }^{* *}, \mathrm{P}<0.001$. LncRNA-CCHE1, cervical carcinoma high-expressed long non-coding RNA 1; PTC,

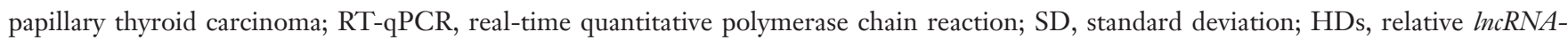
CCHE1 expression; lnc CCHE1, over-expressing lncRNA-CCHE1; sh-CCHE1, knockdown lncRNA-CCHE1.

1:1,000 dilutions of the following primary antibodies: anti-ERK (ab32537), anti-p-ERK (ab176660), anti-p38 MAPK (ab197348), anti-p-p38 MAPK (ab176664), and anti-microtubule protein (ab11304) (Abcam, USA). Then, the membranes were incubated with a 1:2,000 dilution of anti-rabbit or anti-mouse secondary antibody conjugated with horseradish peroxidase (Santa Cruz Biotechnology, Dallas, TX, USA). Image J software (National Institutes of Health, Bethesda, MD, USA) was used to detect the protein expression.

\section{Statistical analysis}

All $\mathrm{P}$ values were determined using GraphPad Prism 7 (GraphPad Software, Inc., San Diego, CA, USA). Data were reported as mean \pm standard deviation $(\mathrm{SD})$. A paired $t$-test was used to compare two-sample means; multiple- sample means were compared using complete randomized block one-way analysis of variance (ANOVA). A P value $<0.05$ indicated a statistically significant difference.

\section{Results}

\section{LncRNA-CCHE1 was bighly expressed in PTC cell lines and tissues and was related to the clinical factors of patients with PTC}

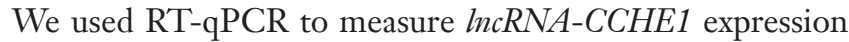
in 51 PTC tissues and adjacent tissues. According to the median value, they were divided into a high expression group and low expression group. The expression of $\operatorname{lnc} R N A$ CCHE1 was higher in the PTC group than in the adjacent tissues (Figure 1A).

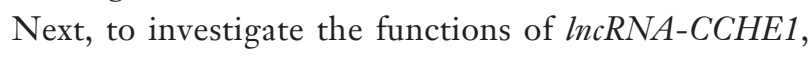


Table 1 Relationship between clinicopathological features and lncRNA-CCHE1 expression in PTC

\begin{tabular}{|c|c|c|c|c|}
\hline \multirow[t]{2}{*}{ Factors } & \multirow[t]{2}{*}{ Number } & \multicolumn{2}{|c|}{$\begin{array}{c}\text { LncRNA-CCHE1 } \\
\text { expression }\end{array}$} & \multirow[t]{2}{*}{$P$ value } \\
\hline & & High & Low & \\
\hline Total & 51 & 26 & 25 & \\
\hline Gender & & & & 0.948 \\
\hline Male & 9 & 4 & 5 & \\
\hline Female & 42 & 22 & 20 & \\
\hline Age (years) & & & & 0.378 \\
\hline$<55$ & 38 & 18 & 20 & \\
\hline$\geq 55$ & 13 & 8 & 5 & \\
\hline Tumor size & & & & 0.488 \\
\hline$<1$ & 24 & 11 & 13 & \\
\hline$\geq 1$ & 27 & 15 & 12 & \\
\hline Tumor numk & & & & $0.025^{\star}$ \\
\hline Solitary & 33 & 13 & 20 & \\
\hline Multiple & 18 & 13 & 5 & \\
\hline \multicolumn{2}{|c|}{ Extrathyroidal extension } & & & $0.017^{*}$ \\
\hline Negative & 24 & 8 & 16 & \\
\hline Positive & 27 & 18 & 9 & \\
\hline \multicolumn{2}{|c|}{ Lymph nodes metastasis } & & & 0.492 \\
\hline Negative & 22 & 10 & 12 & \\
\hline Positive & 29 & 16 & 13 & \\
\hline TNM stage & & & & $0.043^{*}$ \\
\hline I & 41 & 18 & 23 & \\
\hline II-III & 10 & 8 & 2 & \\
\hline BrafV600E & & & & 0.061 \\
\hline$(-)$ & 9 & 2 & 7 & \\
\hline$(+)$ & 42 & 24 & 18 & \\
\hline
\end{tabular}

*, statistically significant $(\mathrm{P}<0.05)$. PTC, papillary thyroid carcinoma; IncRNA-CCHE1, cervical carcinoma high-expressed long non-coding RNA 1; TNM, tumor, node, metastasis.

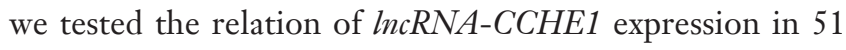
samples with eight clinicopathological parameters, including gender, age, tumor size, tumor number, extrathyroidal extension, lymph node metastasis, tumor, node, metastasis (TNM) stage, and BrafV600E. We evaluated the clinical

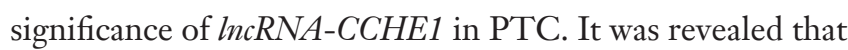

IncRNA-CCHE1 expression was positively correlated with the number of tumors $(\mathrm{P}=0.025)$, extra-glandular invasion $(\mathrm{P}=0.017)$, and TNM stage $(\mathrm{P}=0.043)$ (Table 1$)$, thus suggesting that the abnormal expression of $\operatorname{lncRNA-CCHE1}$ may be involved in PTC pathogenesis.

Then, performed RT-qPCR explore the pattern and level

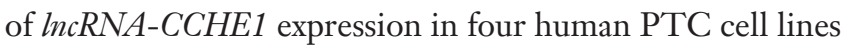
(TPC-1, K1, FTC-133, BCPAP) (Figure 1B). According to our plan to explore whether overexpression of $\operatorname{lnc} R N A$ CCHE1 may be involved in PTC pathogenesis and observe

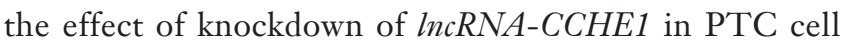
lines, we selected the BCPAP cell line for the following experiment.

\section{LncRNA-CCHE1 is bighly expressed in PTC cell lines}

In this study, BCPAP was selected to examine the role of IncRNA-CCHE1 in PTC lines in vitro. Compared with the non-sense transfection group and the blank control group, lncRNA-CCHE1 expression was increased in the IncRNACCHE1-overexpression group and decreased in the lncRNACCHE1-knockdown group $(\mathrm{P}<0.05)$ (Figure $1 C)$. These results verified that transfection was reliable and effective.

\section{LncRNA-CCHE1 regulated cell proliferation and invasion}

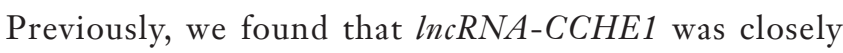
related to the number of tumors and extra-glandular

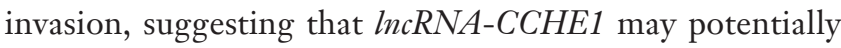
be related to the proliferation and invasion of PTC. Based on these assumptions, we further examined whether IncRNA-CCHE1 could regulate cell proliferation and invasion in vitro. The expression of IncRNA-CCHE1 in BCPAP was both knocked down and overexpressed. The results showed that compared with the blank control group and non-sense transfection group, cell viability of IncRNACCHE1-knockdown BCPAP cells was significantly reduced, while the proliferation of BCPAP cells $\operatorname{lnc} C C H E 1$ was significantly increased (Figure $2 A$ ). In addition, the colony formation assay showed that the colony formation rate was significantly lower in the lncRNA-CCHE1-knockdown group compared to the blank control group and non-sense transfection group, while that of the lncRNA-CCHE1overexpression group was significantly higher compared to the controls (Figure 2B,2C). These results suggest that $\operatorname{lnc} R N A-C C H E 1$ may regulate the proliferation and clonal expansion of PTC cells.

Next, a transwell assay was used to evaluate the invasion 
A

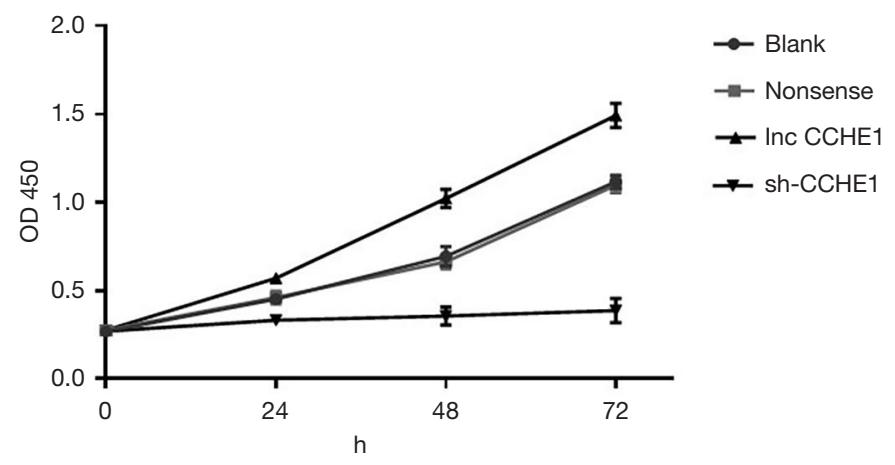

B

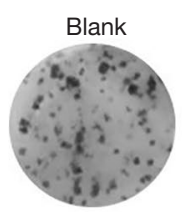
Inc CCHE1

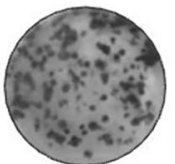

D

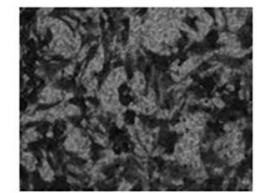

Inc CCHE1

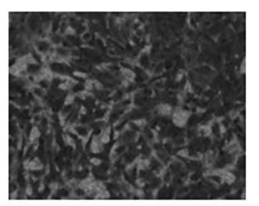

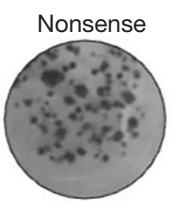

sh-CCHE1

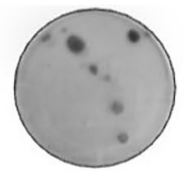

Nonsense

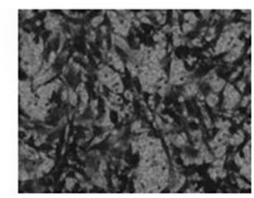

sh-CCHE1

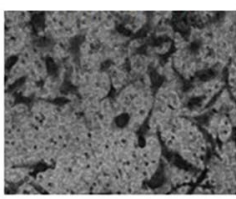

C
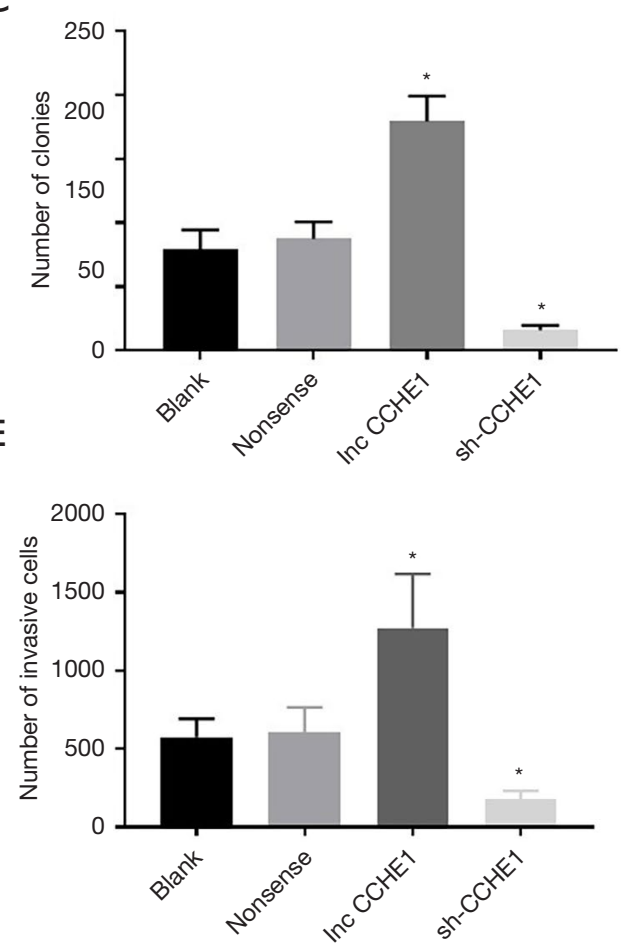

Figure 2 The effects of lnc CCHE1 and sh-CCHE1 on BCPAP cell proliferation, cell invasion and migration in vitro. (A) Proliferation of BCPAP cells was detected in the blank, non-sense, lnc CCHE1, and sh-CCHE1 transfected cells using the CCK-8 assay. (B,C) Colony formation also was detected in the blank, non-sense, lnc CCHE1, and sh-CCHE1 transfected cells. (D,E) A transwell assay was used to assess the invasion ability in blank, non-sense, lnc CCHE1, and sh-CCHE1 transfected cells. (B) Take photos with a camera. (D) Scale bar: $200 \mu \mathrm{m}$. (B,D) Dye with crystal violet. Data in (B,E) are means \pm SDs of three independent experiments using PTC cells from different HDs. *, P<0.05. LncRNA-CCHE1, cervical carcinoma high-expressed long non-coding RNA 1; siRNA, small interfering RNA; CCK-8, Cell Counting Kit-8; shRNA, short hairpin RNA; SD, standard deviation; PTC, papillary thyroid carcinoma; HDs, number of cells; OD, optical density; lnc CCHE1, over-expressing lncRNA-CCHE1; sh-CCHE1, knockdown lncRNA-CCHE1.

ability of PTC cell lines. Compared with the non-sense transfection group and the blank control, $\operatorname{lncRNA}$-CCHE1 knockdown inhibited cell invasion, while $\operatorname{lncRNA-CCHE1}$ overexpression improved cell viability (Figure 2D,2E). Taken together, these results confirm that $\ln c \mathrm{RNA}$-CCHE1 may be involved in regulating the invasion and proliferation of PTC cells.

\section{LncRNA-CCHE1 regulated cell apoptosis}

Flow cytometry was used to assess apoptosis in PTC cell lines. Results revealed that, compared with the non-sense 
A

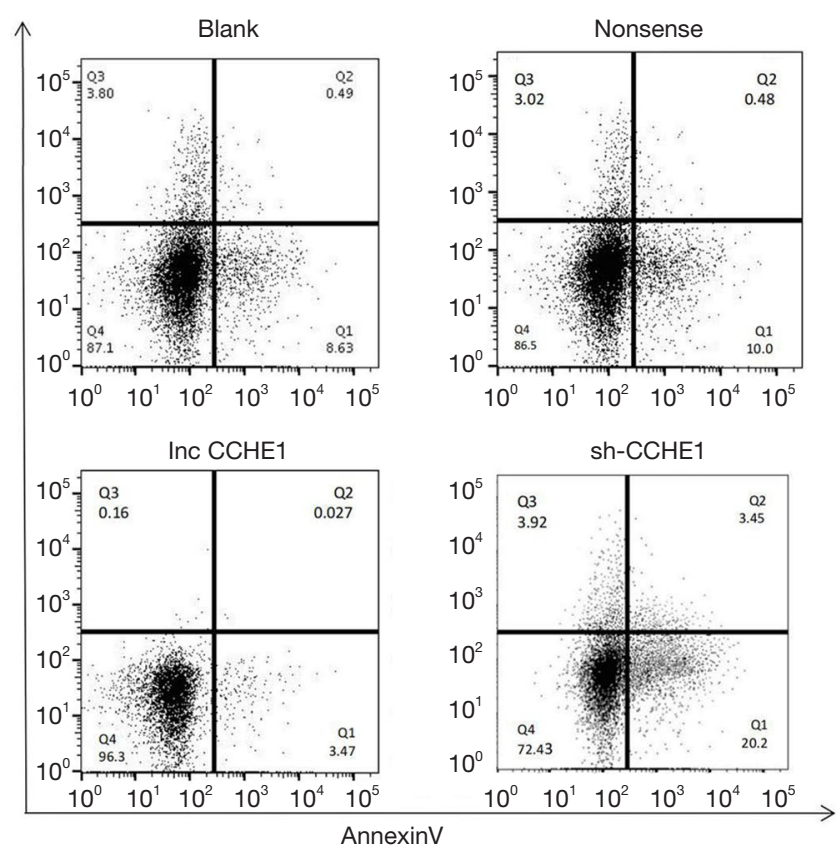

B

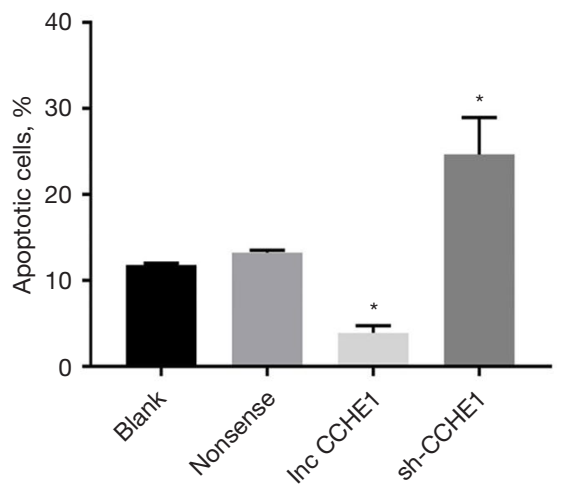

Figure 3 The effects of lnc CCHE1 and sh-CCHE1 on BCPAP cell apoptosis in vitro. (A,B) Flow cytometry test was used to detect apoptosis in the blank, non-sense, lnc CCHE1, and sh-CCHE1 transfected cells. Data in (B) are means \pm SDs of three independent experiments using BCPAP cells from different HDs. *, $\mathrm{P}<0.05$. LncRNA-CCHE1, cervical carcinoma high-expressed long non-coding RNA 1; shRNA, short hairpin RNA; SD, standard deviation; PI, propidium iodide; HDs, apoptotic cells percentage; lnc CCHE1, over-expressing IncRNA-CCHE1; sh-CCHE1, knockdown lncRNA-CCHE1.

transfection group and the blank control group, $\operatorname{lnc} R N A$ CCHE1 knockdown increased cell apoptosis, while $\operatorname{lnc} R N A$ CCHE1 over-expression inhibited apoptosis $(\mathrm{P}<0.05)$

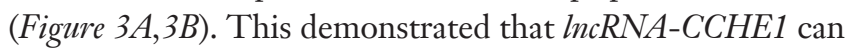
regulate cell apoptosis in PTC cell lines.

\section{LncRNA-CCHE1 influences the tumorigenicity of PTC cells via the ERK/MAPK patbway}

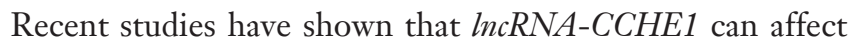
liver cancer (22) and lung cancer progression (26) through the ERK/MAPK pathway. The ERK/MAPK pathway is one of the most classical signal transduction pathways, through which several key proto-oncogenes and growth factors are mediated to promote tumor growth. In this study, we found that the down-regulation or up-regulation of $\operatorname{lnc} R N A$ CCHE1 may alter the phosphorylation of ERK and P38 MARK in PTC cell lines (Figure 4A); down-regulation of lncRNA-CCHE1 expression in BCPAP cells led to decreased phosphorylation of ERK and MAPK, whereas up-regulation

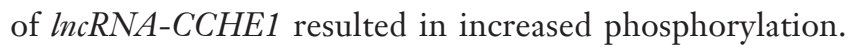

At the same time, gray value analysis was carried out for the results, and the results were consistent (Figure 4B).

\section{Discussion}

Accumulating discoveries suggested that lncRNAs play functional roles in TC, including PTCSC3 (27), MALAT1 (28), MEG3 (29), NEAT1 (30), and so on. Among them, PTC susceptibility candidate 3 (PTCSC3)/miR-574-5p mediated the proliferation and migration of PTC-1 cells through regulating the activity of $\mathrm{Wnt} / \beta$-catenin (27). Nuclear enrich abundant transcript 1 (NEAT1) promoted the both onset and the malignant progression of TC through regulating miRNA-214 expression (30). All of these have confirmed that the possibility of IncRNAs serve as

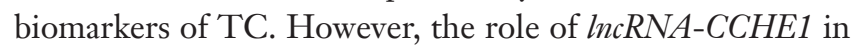
PTC has rarely reported.

We found that $\ln R N A-C C H E 1$ was highly expressed in PTC cell lines and tissues. Combined with the clinicopathological characteristics, we also discovered that lncRNA-CCHE1 expression was positively correlated with 
A

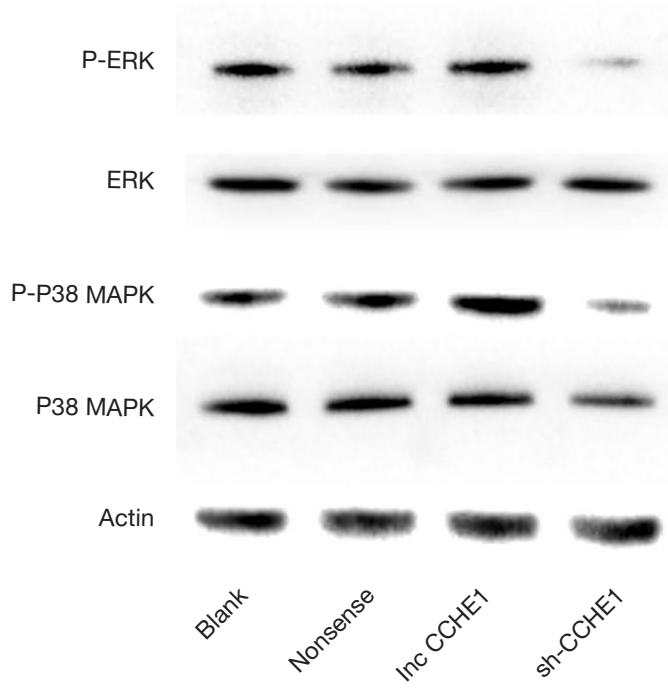

B
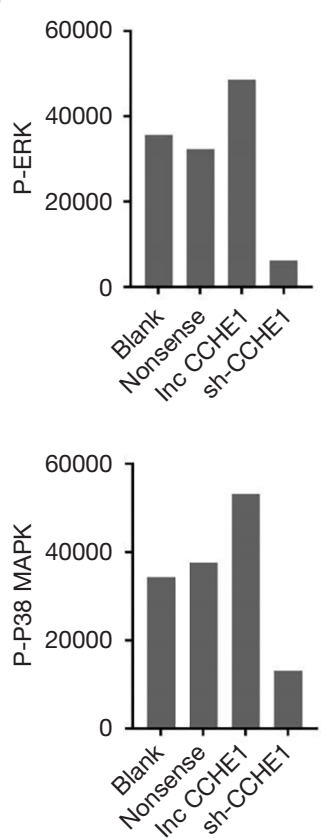
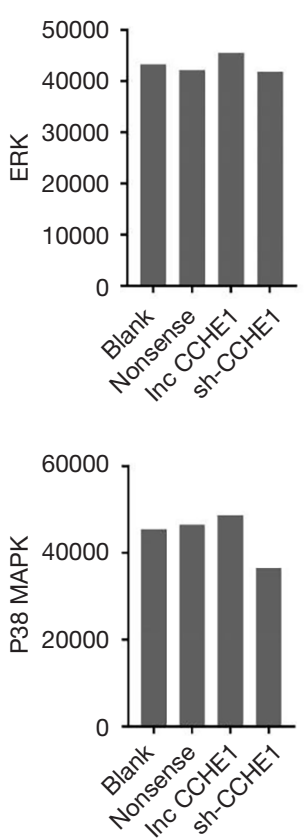

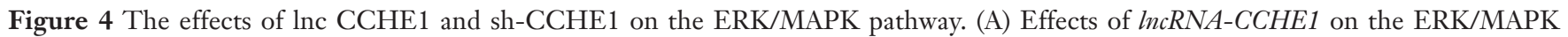
pathway as detected by western blotting. Changes in ERK and p38 MAPK activation were observed in the blank, non-sense, lnc CCHE1, and sh-CCHE1 transfected cells. (B) Gray value results. The experiments were performed in triplicate. LncRNA-CCHE1, cervical carcinoma high-expressed long non-coding RNA 1; ERK/MAPK, extracellular signal-regulated kinase/mitogen-activated protein kinase; lnc CCHE1,

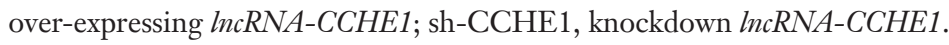

tumor number, extra-glandular invasion, and TNM stage. In principle, for patients with extra-glandular invasion and multifocal lesions, clinicians will take a more aggressive surgical approach. When considered together with version 8 of the American Joint Committee on Cancer (AJCC) TNM staging system, tumors with multifocal, extra-glandular invasion would predict a later stage and poorer prognosis. Therefore, we thought that the high expression of $\operatorname{lnc} R N A$ CCHE1 would play an important role on the development of PTC, which would have the potential to be a molecular marker to detect the malignancy of thyroid cancer. Of course, this requires further study in the later stage, as well as the accumulation and testing of clinical samples. In addition, we suspected that there might be a relationship between BrafV600E mutation and IncRNA-CCHE1, although the statistical results showed no correlation. We think this may have been related to the sample size. These

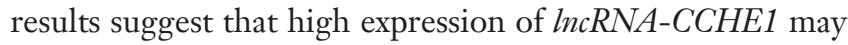
participate in the pathogenesis of PTC by promoting cancer development. In addition, our results verified that $\ln c R N A$ CCHE1 has an indispensable role in the proliferation and apoptosis of PTC cells in vitro.

Previous studies have confirmed that lncRNAs are involved in tumorigenesis, as well as in the treatment and prognosis of tumors (17,31-33). For example, Xu et al.

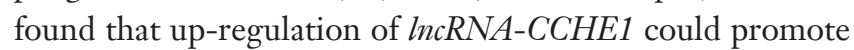
gastric cancer cell proliferative ability and colony formation, inhibiting cell apoptosis; however, low-expression of

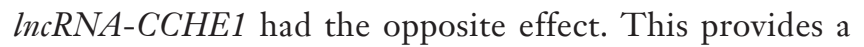
basis for the application of this factor in the diagnosis of gastric cancer (17). Wang et al. found that knockdown of IncRNA-CCHE1 curbed the proliferation, migration, and invasion and hastened the apoptosis in oral squamous cell carcinoma (OSCC) cell lines (19). Some scholars (34-37) have showed that lncRNAs may directly bind protens, microRNAs, or mRNAs and alter the activity or/and expression of them to regulate the gene expressions. In this study, we found that the inhibition of $\operatorname{lncRNA-CCHE1}$ reduced PTC cell proliferation and invasion, while its overexpression caused the opposite effect. Meanwhile, $\operatorname{lncRNA-}$ CCHE1 knockdown increased apoptosis when compared to the overexpression group, indicating that $\operatorname{lncRNA-CCHE1}$ 
is also involved in apoptosis in PTC cells. Previous studies have shown that dysregulation of lncRNAs may also lead to tumor progression and uncontrolled growth by altering additional pathways (38). Thus we considered that $\ln R N A$ CCHE1 may regulate the proliferation, migration, and invasion of PTC cells through some pathways.

Previous studies have shown that the ERK/MAPK pathway has an important role in different cancers. Liao et al. discovered that through the ERK/MAPK pathway IncRNA-CCHE1 promoted the proliferation, migration, and invasion ability of non-small cell lung cancer (NSCLC) cell line (26). Zhang et al. found that GINS2 also inhibits cell activity by interfering with the MAPK/ERK pathway and induces cell cycle arrest, thus promoting apoptosis of pancreatic cancer cells (39). Moreover, Du et al. showed that inhibition of $S L C 25 A 22$ by the MAPK/ERK pathway could promote mitochondrial apoptosis, thus inhibiting the growth and proliferation gallbladder carcinoma cells (40).

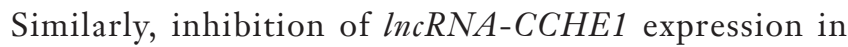
BCPAP cells resulted in decreased phosphorylation of ERK and MAPK, whereas overexpression of $\operatorname{lncRNA-CCHE1}$ resulted in increased phosphorylation. However, no changes were detected in total ERK and MAPK protein expression. Therefore, at least part of the explanation for the changes in cell biological function caused by $\ln c R N A-C C H E 1$ knockdown in vitro PTC growth cells can be explained by the inhibition of the ERK/MAPK pathway. However, the

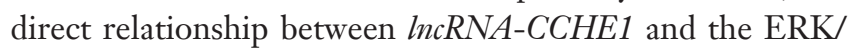
MAPK pathway requires further investigation.

Altogether, the presented results may introduce a key oncogene as a new potential diagnostic target for PTC patients. In previous studies, NSCLC patients with high $\operatorname{lnc} R N A$-CCHE1 expression showed a worse prognosis when

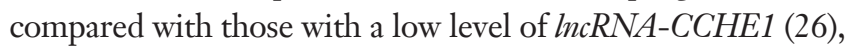
and this was the same in cervical cancer (27) and hepatocellular carcinoma (22). Our findings may also be of value to predict the poor prognosis PTC patients; however, this needs to be further investigated in studies with a larger sample size and longer follow-up.

This study only confirmed the potential significance of lncRNA-CCHE1 in PTC. At the same time, we provided basic evidence to support targeted therapies that inhibit

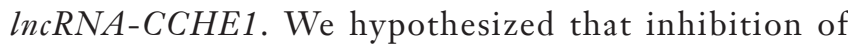
lncRNA-CCHE1 targeted therapy might contribute to the control of PTC, as $\ln c R N A-C C H E 1$ could modulate the important pathways in PTC. This is useful for $\operatorname{lnc} R N A$ CCHE1 directed diagnostic and therapeutic of strategy against PTC.
However, there are some limitations in our research. On the one hand, some experiments in vivo experiments have not been increased. On the other hand, $\operatorname{lncRNA} A$ CCHE1 through the signal pathway of ERK/MAPK play an important role in PTC, but the effect of inhibitors or agonists was not be added to certified the mechanism. In the future, we will need to further investigate in these aspects.

\section{Conclusions}

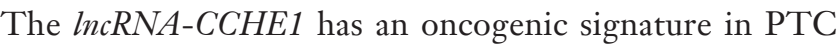
cells. It also has a role in the pathogenesis of TC and may potentially be used as biomarker to evaluate whether TC patients require surgery and early intervention. These data provide a theoretical basis for early clinical diagnosis and treatment of PTC.

\section{Acknowledgments}

The authors thank the patients and healthy volunteers for their cooperation and participation in the study.

Funding: This study was supported by the Hebei Science and Technology Planning grant (16967788D) and Department of Health of Hebei Province grant (20190085).

\section{Footnote}

Reporting Checklist: The authors have completed the MDAR reporting checklist. Available at https://dx.doi. org/10.21037/tcr-21-1502

Data Sharing Statement: Available at https://dx.doi. org/10.21037/tcr-21-1502

Conflicts of Interest: All authors have completed the ICMJE uniform disclosure form (available at https://dx.doi. org/10.21037/tcr-21-1502). The authors have no conflicts of interest to declare.

Ethical Statement: The authors are accountable for all aspects of the work in ensuring that questions related to the accuracy or integrity of any part of the work are appropriately investigated and resolved. All procedures performed in this study involving human participants were in accordance with the Declaration of Helsinki (as revised in 2013). This study was approved by the Ethics Committee of Hebei Medical University Fourth Affiliated Hospital (2020KS018). All participants fully understood the 
experimental protocol and signed informed consent forms. Patient consent for publication is not applicable.

Open Access Statement: This is an Open Access article distributed in accordance with the Creative Commons Attribution-NonCommercial-NoDerivs 4.0 International License (CC BY-NC-ND 4.0), which permits the noncommercial replication and distribution of the article with the strict proviso that no changes or edits are made and the original work is properly cited (including links to both the formal publication through the relevant DOI and the license). See: https://creativecommons.org/licenses/by-nc-nd/4.0/.

\section{References}

1. Valvo V, Nucera C. Coding molecular determinants of thyroid cancer development and progression. Endocrinol Metab Clin North Am 2019;48:37-59.

2. Ho AS, Luu M, Barrios L, et al. Incidence and mortality risk spectrum across aggressive variants of papillary thyroid carcinoma. JAMA Oncol 2020;6:706-13.

3. Haugen BR. 2015 American Thyroid Association management guidelines for adult patients with thyroid nodules and differentiated thyroid cancer: what is new and what has changed? Cancer 2017;123:372-81.

4. Yan HX, Du J, Fu J, et al. Microarray-based differential expression profiling of long noncoding RNAs and messenger RNAs in formalin-fixed paraffin-embedded human papillary thyroid carcinoma samples. Transl Cancer Res 2019;8:439-51.

5. Tsai MC, Manor O, Wan Y, et al. Long noncoding RNA as modular scaffold of histone modification complexes. Science 2010;329:689-93.

6. Ponting CP, Oliver PL, Reik W. Evolution and functions of long noncoding RNAs. Cell 2009;136:629-41.

7. Kashi K, Henderson L, Bonetti A, et al. Discovery and functional analysis of lncRNAs: Methodologies to investigate an uncharacterized transcriptome. Biochim Biophys Acta 2016;1859:3-15.

8. Gamaev L, Mizrahi L, Friehmann T, et al. The prooncogenic effect of the lncRNA H19 in the development of chronic inflammation-mediated hepatocellular carcinoma. Oncogene 2021;40:127-39.

9. Yu L, Huo L, Shao X, et al. lncRNA SNHG5 promotes cell proliferation, migration and invasion in oral squamous cell carcinoma by sponging miR-655-3p/FZD4 axis. Oncol Lett 2020;20:310.

10. Jiang $X$, Ye Z, Jiang $Y$, et al. LncRNA OIP5-AS1 upregulates snail expression by sponging miR-34a to promote ovarian carcinoma cell invasion and migration. Biol Res 2020;53:49.

11. Zhao ZB, Chen F, Bai XF. Long noncoding RNA MALAT1 regulates hepatocellular carcinoma growth under hypoxia via sponging microRNA-200a. Yonsei Med J 2019;60:727-34.

12. Xu Y, Zhang $\mathrm{X}, \mathrm{Hu} \mathrm{X}$, et al. The effects of lncRNA MALAT1 on proliferation, invasion and migration in colorectal cancer through regulating SOX9. Mol Med 2018;24:52.

13. Shen F, Zheng H, Zhou L, et al. Overexpression of MALAT1 contributes to cervical cancer progression by acting as a sponge of miR-429. J Cell Physiol 2019;234:11219-26.

14. Cao B, Liu C, Yang G. Down-regulation of lncRNA ADAMTS9-AS2 contributes to gastric cancer development via activation of PI3K/Akt pathway. Biomed Pharmacother 2018;107:185-93.

15. Wang A, Jin C, Li H, et al. LncRNA ADAMTS9-AS2 regulates ovarian cancer progression by targeting miR182-5p/FOXF2 signaling pathway. Int J Biol Macromol 2018;120:1705-13.

16. Shi YF, Lu H, Wang HB. Downregulated lncRNA ADAMTS9-AS2 in breast cancer enhances tamoxifen resistance by activating microRNA-130a-5p. Eur Rev Med Pharmacol Sci 2019;23:1563-73.

17. Xu G, Zhang Y, Li N, et al. LncRNA CCHE1 in the proliferation and apoptosis of gastric cancer cells. Eur Rev Med Pharmacol Sci 2018;22:2631-7.

18. Jin X, Ye L, Lin M, et al. lncRNA-CCHE1 is involved in migration and invasion but not in proliferation of pancreatic adenocarcinoma cells possibly by interacting with ROCK1. Oncol Lett 2019;18:1218-24.

19. Wang $\mathrm{Y}$, Tong J, Lin H, et al. CCHE1 accelerated the initiation of oral squamous cell carcinoma through enhancing PAK2 expression by sponging miR-922. J Oral Pathol Med 2020;49:636-44.

20. Zhang Z, Yu T, Geng W. Long non-coding RNA CCHE1 participates in postoperative distant recurrence but not local recurrence of osteosarcoma possibly by interacting with ROCK1. BMC Musculoskelet Disord 2020;21:462.

21. Yang M, Zhai X, Xia B, et al. Long noncoding RNA CCHE1 promotes cervical cancer cell proliferation via upregulating PCNA. Tumour Biol 2015;36:7615-22.

22. Peng $W$, Fan H. Long noncoding RNA CCHE1 indicates a poor prognosis of hepatocellular carcinoma and promotes carcinogenesis via activation of the ERK/MAPK 
pathway. Biomed Pharmacother 2016;83:450-5.

23. Mc'Tiernan AM, Weiss NS, Daling JR. Incidence of thyroid cancer in women in relation to reproductive and hormonal factors. Am J Epidemiol 1984;120:423-35.

24. Derwahl M, Nicula D. Estrogen and its role in thyroid cancer. Endocr Relat Cancer 2014;21:T273-83.

25. Hima S, Sreeja S. Modulatory role of $17 \beta$-estradiol in the tumor microenvironment of thyroid cancer. IUBMB Life 2016;68:85-96.

26. Liao Y, Cheng S, Xiang J, et al. lncRNA CCHE1 increased proliferation, metastasis and invasion of nonsmall lung cancer cells and predicted poor survival in nonsmall lung cancer patients. Eur Rev Med Pharmacol Sci 2018;22:1686-92.

27. Fan M, Li X, Jiang W, et al. A long non-coding RNA, PTCSC3, as a tumor suppressor and a target of miRNAs in thyroid cancer cells. Exp Ther Med 2013;5:1143-6.

28. Huang JK, Ma L, Song WH, et al. LncRNA-MALAT1 promotes angiogenesis of thyroid cancer by modulating tumor-associated macrophage FGF2 protein secretion. J Cell Biochem 2017;118:4821-30.

29. Wang C, Yan G, Zhang Y, et al. Long non-coding RNA MEG3 suppresses migration and invasion of thyroid carcinoma by targeting of Rac1. Neoplasma 2015;62:541-9.

30. Li JH, Zhang SQ, Qiu XG, et al. Long non-coding RNA NEAT1 promotes malignant progression of thyroid carcinoma by regulating miRNA-214. Int J Oncol 2017;50:708-16.

31. Ni W, Zhang Y, Zhan Z, et al. A novel lncRNA uc.134 represses hepatocellular carcinoma progression by inhibiting CUL4A-mediated ubiquitination of LATS1. J Hematol Oncol 2017;10:91.

32. Song J, Chen X, Tian Q, et al. The value of lncRNA GHET1 as a prognostic factor for survival of Chinese cancer outcome: a meta-analysis. Dis Markers

Cite this article as: Shi P, Liu Y, Zhang M, Yang J, Jing S, Yang D, Liu F, Wu Y, Shi H, Geng C. Cervical carcinoma highexpressed long non-coding RNA 1 promotes papillary thyroid carcinoma cell proliferation and invasion. Transl Cancer Res 2021;10(9):4158-4168. doi: 10.21037/tcr-21-1502
2019;2019:5824190.

33. Zhou X, Fan Y, He Y, et al. Clinicopathological and prognostic value of gastric carcinoma highly expressed transcript 1 in cancer: a meta-analysis. J Oncol 2020;2020:6341093.

34. Quagliata L, Matter MS, Piscuoglio S, et al. Long noncoding RNA HOTTIP/HOXA13 expression is associated with disease progression and predicts outcome in hepatocellular carcinoma patients. Hepatology 2014;59:911-23.

35. Xu Y, Wang J, Qiu M, et al. Upregulation of the long noncoding RNA TUG1 promotes proliferation and migration of esophageal squamous cell carcinoma. Tumour Biol 2015;36:1643-51.

36. Salmena L, Poliseno L, Tay Y, et al. A ceRNA hypothesis: the Rosetta Stone of a hidden RNA language? Cell 2011;146:353-8.

37. Cesana M, Cacchiarelli D, Legnini I, et al. A long noncoding RNA controls muscle differentiation by functioning as a competing endogenous RNA. Cell 2011;147:358-69.

38. Xia F, Xia W, Yu X. LncRNA HOTAIR influences the growth, migration, and invasion of papillary thyroid carcinoma via affection on the miR-4885p/NUP205 axis. Technol Cancer Res Treat 2020;19:1533033820962125.

39. Zhang M, He S, Ma X, et al. GINS2 affects cell viability, cell apoptosis, and cell cycle progression of pancreatic cancer cells via MAPK/ERK pathway. J Cancer 2020;11:4662-70.

40. Du P, Liang H, Fu X, et al. SLC25A22 promotes proliferation and metastasis by activating MAPK/ERK pathway in gallbladder cancer. Cancer Cell Int 2019;19:33.

(English Language Editor: J. Jones) 